\title{
$B$-BOUNDED SEMIGROUPS AND IMPLICIT EVOLUTION EQUATIONS
}

\author{
J. BANASIAK
}

Received 6 April 2000

The aim of this paper is to show an application of the recently introduced $B$-bounded semigroups in the theory of implicit and degenerate evolution equations. The most interesting feature of this approach is its applicability to problems with noncloseable operators.

\section{Introduction}

Consider the Cauchy problem for the implicit evolution equation

$$
\frac{d}{d t}(K u)=L u, \quad \lim _{t \rightarrow 0^{+}}(K u)(t)=\stackrel{\circ}{u},
$$

where $K: Z \rightarrow X, L: Z \rightarrow X, Z, X$ are, say, Banach spaces, and $K, L$ are linear operators. There is a number of approaches to solving such problems; for example, $[11,12]$, similar in spirit the results of $[15,16,17,18]$ where an interesting notion of empathy is introduced, or [20] where a suitable change of space method is used. In this paper, we aim neither at a comprehensive treatment of the problem (1.1), nor at an exhaustive comparison of various methods employed to solve it, but we rather describe how a new notion of $B$-bounded semigroups, introduced in $[8,10]$ and investigated in $[3,4]$, can be used in this field.

One of the "natural" ways of approaching (1.1) would be to factor out $K$ and, provided it is invertible, to consider a standard Cauchy problem with the operator $K^{-1} L$ on the right-hand side. In some cases, however, the operator $K$ is not closeable and therefore there is no way the "time derivative" or the limit at $t=0^{+}$can commute with $K$. Thus, it is reasonable to study $(1.1)$ as it stands. In [18, 19] this problem is treated by introducing a pair of evolution families, called an empathy. As we will see, the theory of $B$-bounded semigroups provides another convenient way of performing this "impossible" commutations by passing to a specially constructed space related to $K$. In this paper, a similar approach to that of [20] is used but some adopted assumptions on $K$ are less restrictive.

Copyright $\odot 2000$ Hindawi Publishing Corporation

Abstract and Applied Analysis 5:1 (2000) 13-32

2000 Mathematics Subject Classification: 47D06, 34G10

URL: http://aaa.hindawi.com/volume-5/S1085337500000087.html 
It is worthwhile to note that the method of $B$-bounded semigroups does not require $X$ to be a Banach space (in fact $X$ is not required to have any structure but linear) and consequently the operators $K$ and $L$ are not assumed to have any standard topological properties when considered separately; we require, however, their good behaviour in the abstract extrapolation space $X_{B}$ introduced in [4] or, equivalently, a good behaviour of the operator $K^{-1} L$ (or its suitable realization) in $Z$. The idea is similar to that of $[7,14]$, where the authors also seek a modification of the original space in which the given operators are, for example, closeable. Our method on one hand is less general, as the modified space is defined in a prescribed way by the operators appearing in the problem, but on the other hand this space may be much less restrictive than that stipulated in the work cited.

As we mentioned before, we do not give a survey of all available methods for solving (1.1); instead we demonstrate links between $B$-bounded semigroups and the empathy theory which is also focused on solving problems with possibly noncloseable $K$, and with the method employed by Showalter in [20], which seems to be a particular case of the $B$-bounded semigroup method.

To keep the exposition within a reasonable length we focus on linear operators with $K$ invertible. A generalization to multivalued and nonlinear cases can be done along the lines of, for example, $[11,12,15,20]$ with only minor difficulties (see also Remark 3.8). We intend to pursue this topic provided interesting applications arise.

It is also worthwhile to note that despite superficial similarities of $B$-bounded semigroups and $C$-existence families, these two notions coincide only for a very restricted class of operators. This question is addressed in detail in [5].

\section{2. $B$-bounded semigroups revisited}

We start with recalling basic facts from the theory of $B$-bounded semigroups and give generalizations relevant to the theory of implicit evolution equations.

We consider the standard abstract Cauchy problem in a Banach space $X$ :

$$
\frac{d u}{d t}=A u, \quad \lim _{t \rightarrow 0^{+}} u(t)=\stackrel{\circ}{u} .
$$

Very often the existence of the semigroup $(\exp (t A))_{t \geq 0}$ describing the evolution of the system is established in a nonconstructive way. This is especially the case when the positivity methods are employed (cf. [1]). Then very little quantitative information on the evolution is available. On the other hand, there may exist an operator $B$ such that $t \rightarrow B e^{t A}$ can be calculated constructively yielding some information about the evolution. An example of this type, pertaining to the transport equation with multiplying boundary conditions, was analysed in [10] and has prompted one of the authors to define a class of evolution families which behave well if looked at through the "lenses" of another operator, and which can be thought of as generalizations of $\left\{B e^{t A}\right\}_{t \geq 0}$. Such families, called $B$-bounded semigroups, have been introduced in [8], and analysed and applied to various problems in a few papers $[3,4,6,9]$.

The definition of $B$-quasi bounded semigroups as introduced in [9] (with some modifications due to the author of this paper) reads as follows. 
Definition 2.1. Let $(A, D(A))$ be a linear operator in a Banach space $X$ and $(B, D(B))$ be another linear operator from $X$ to another Banach space $Z$ with $D(A) \subset D(B)$, and let for some $\omega \in \mathbb{R}$ the resolvent set of $A$ satisfies

$$
\rho(A) \supset] \omega, \infty[.
$$

A one-parameter family of operators $(Y(t))_{t \geq 0}$ from $X$ to $Z$, which satisfies

(1) $D(Y(t))=: \Omega \supseteq D(B)$, and for any $t \geq 0$ and $f \in D(B)$

$$
\|Y(t) f\|_{Z} \leq M \exp (\omega t)\|B f\|_{Z},
$$

(2) the function $t \rightarrow Y(t) f \in C([0, \infty[, Z)$ for any $f \in \Omega$,

(3) for any $f \in \Omega_{0}:=\{f \in D(A) \cap D(B) ; A f \in \Omega\} \subset D(A) \cap D(B)$

$$
Y(t) f=B f+\int_{0}^{t} Y(s) A f d s, \quad t \geq 0
$$

is called a $B$-quasi bounded semigroup generated by $A$.

To shorten notation, if $A$ generates a $B$-bounded semigroup satisfying the above conditions, then we write $A \in B-\mathscr{G}(M, \omega, X, Z)$. We also shorten the name saying that the family $(Y(t))_{t \geq 0}$ defined in Definition 2.1 is a $B$-bounded semigroup generated by $A$. We also use the standard notation $A \in \mathscr{G}(M, \omega, X)$ to express the fact that $A$ is the generator of a $C_{0}$-semigroup in $X$ with the Hille-Yosida constants $M$ and $\omega$.

Remark 2.2. It follows that the assumptions [3]: $D(A) \subset D(B)$ and (2.2) can be replaced by a single assumption that for $\lambda>\omega$ the operator

$$
(\lambda I-A): D_{B}(A) \longrightarrow D(B),
$$

where

$$
D_{B}(A)=\{x \in D(A) \cap D(B) ; A x \in D(B)\}
$$

is bijective. Note that this requirement is purely algebraic.

We recall here the main results of [4] together with some recent generalizations due to [3].

The main role in the considerations of [4] is played by the space $X_{B}$ which is the completion of the quotient space $D(B) / N(B)$ with respect to the seminorm $\|\cdot\|_{B}=$ $\|B \cdot\|_{Z}$. It is known that then $D(B) / N(B)$ is isometrically isomorphic to a dense subspace of $X_{B}$, say, $\mathscr{X}$. The canonical injection of $X$ into $X_{B}$ (and onto $\mathscr{X}$ ) is denoted by $\mathfrak{p}$. In a standard way $B$ extends by density to an isometry $\mathfrak{B}: X_{B} \rightarrow Z$.

An important observation is that if $A$ generates a $B$-bounded semigroup, then $A$ preserves cosets of $D(B) / N(B)$ and therefore it can be defined to act from $\mathfrak{p} D_{B}(A) \subset \mathscr{X}$ into $\mathscr{X}$. We denote by $A_{B}$ the part of $A$ in $D(B)$, that is, $A_{B}=\left.A\right|_{D_{B}(A)}$. It can be also proved [4] that if $A \in B-\mathscr{G}(M, \omega, X, Z)$, then the shift of $A$ to $\mathscr{X}$ is closeable in $X_{B}$; we denote its closure by $\mathfrak{A}$.

To simplify the notation we use the same notation for the operators $A$ and $B$ defined on $\mathscr{X}$ and their shifts which is possible by [4]; with this convention the injection $\mathfrak{p}$ 
becomes the identity (or more precisely projection) and for any operator $\mathfrak{C}$ defined in $X_{B}$ and $x \in D(B)$, the symbol $\mathfrak{C} x$ is to be understood as $\mathfrak{C} p$, if the latter is defined.

We introduce the subspace $Z_{B}=\overline{R(B)}$ (the closure of the range of $B$ in $Z$ ). The main result of [4, Theorem 4.1], reads as follows.

Theorem 2.3. If $A \in B-\mathscr{G}(M, \omega, X, Z)$ and ${\overline{B\left[D_{B}(A)\right]}}^{Z}=Z_{B}$, then $\mathfrak{A} \in \mathscr{G}\left(M, \omega, X_{B}\right)$. Conversely, if there is $\mathscr{A} \supset A$ such that $\mathscr{A} \in \mathscr{G}\left(M, \omega, X_{B}\right)$, then $\mathscr{A}=\mathfrak{A}$ and $A \in$ $B-\mathscr{G}(M, \omega, X, Z)$.

The B-bounded semigroup $(Y(t))_{t \geq 0}$ for $x \in D(B)$ is given by

$$
Y(t) x=\exp \left(t \mathfrak{B A} \mathfrak{B}^{-1}\right) B x=\mathfrak{B} \exp (t \mathfrak{A}) x .
$$

The assumption that $B\left[D_{B}(A)\right]$ is dense in $Z_{B}$ can be discarded if $Z$ (and consequently $Z_{B}$ ) are reflexive spaces (see [4, Corollary 4.1]). Recently Arlotti [3] proved that if the $B$-bounded semigroup satisfies the additional condition

$$
\forall x \in D(B), Y(0) x=B x,
$$

then $B\left[D_{B}(A)\right]$ is dense in $Z_{B}$ (or equivalently, $D_{B}(A)$ is dense in $X_{B}$ ). Note that (2.4) gives (2.8) only for $x \in \Omega_{0}$ that, in most cases, reduces to $D_{B}(A)$.

It is easy to see that the converse is also true. Therefore, if (2.8) holds, then the density assumption in Theorem 2.3 can be omitted.

Since the space $X_{B}$ is in many cases rather difficult to handle, Theorem 2.3 is most often used in the following version (see [4, Theorem 4.3] and [3, Theorem 2.1]).

THeOREM 2.4. Let the operators $A$ and B satisfy the conditions of Definition 2.1. Then $A$ is the generator of a B-quasi bounded semigroup satisfying (2.8) if and only if the following conditions hold:

(1) $B\left[D_{B}(A)\right]$ is dense in $Z_{B}$,

(2) there exist $M>0$ and $\omega \in \mathbb{R}$ such that for any $x \in D(B), \lambda>\omega$ and $n \in \mathbb{N}$ :

$$
\left\|B(\lambda I-A)^{-n} x\right\|_{Z} \leq \frac{M}{(\lambda-\omega)^{n}}\|B x\|_{Z} .
$$

If we do not require $(Y(t))_{t \geq 0}$ to satisfy (2.8), then condition (1) is sufficient but not necessary.

The main point in the proof of Theorem 2.4 is the observation that (2.9) can be extended to hold on the entire $X_{B}$. This allows a useful corollary.

Corollary 2.5. Let the operators A and B satisfy the conditions of Definition 2.1 and let also assumptions (1) and (2) of Theorem 2.4 be satisfied.

(1) If the estimate (2.9) is satisfied for $n=1$ with $M=1$ and $\omega=0$, then $\mathfrak{A}$ generates a semigroup of contractions in $X_{B}$ and consequently $A \in B-\mathscr{G}(1,0, X, Z)$.

(2) If the estimate

$$
\left\|B(\lambda I-A)^{-1} x\right\|_{Z} \leq \frac{M}{|\lambda-\omega|}\|B x\|_{Z}
$$


holds for $\lambda \in S_{\theta}=\{\lambda \in \mathbb{C} ;|\operatorname{Arg} \lambda| \leq \pi / 2+\theta, \theta>0\}$, then $\mathfrak{A}$ generates an analytic semigroup in $X_{B}$ and consequently $A \in B-\mathscr{G}\left(M^{\prime}, \omega, X, Z\right)$ for some constant $M^{\prime}$.

2.1. Further improvement of the generation theorem. Note that it is not necessary for $A$ to generate a semigroup in $X$. Thus, the existence of a $B$-bounded semigroup is no longer related to the existence of $(\exp (t A))_{t \geq 0}$, as was the case in the motivating example of [8]. As mentioned before, the assumption that $[\omega, \infty[\subseteq \rho(A)$ for some $\omega \in \mathbb{R}$ was replaced by the requirement that $(\lambda-A): D_{B}(A) \rightarrow D(B)$ is bijective. It follows that this assumption can be relaxed even further. A detailed discussion of this topic together with the proofs can be found in [5]. Here we sketch the main results.

Our aim is to replace assumption (2.2) by a weaker one which would require only the bijectivity of a suitable extension of $A$. In fact, in the proof of Theorem 2.3 the assumption (2.2) is used to show that $[\omega, \infty[\subset \rho(\mathfrak{A})$. Thus, what we really need is the Hille-Yosida estimate valid on some dense subspace $\mathfrak{X}$ of $X_{B}$. Moreover, as we are using the pseudo-resolvent identity, we must have that $D_{\lambda}=(\lambda I-A)^{-1} \mathfrak{X} \subset \mathfrak{X}$ for $\lambda>\omega$ and this yields that $D_{\lambda}$ must be independent of $\lambda$ [5]. Finally, as our starting point are $X$ and the operators defined in it, the space $\mathfrak{X}$ must be accessible from $X$ in the sense of the operator closure in $X_{B}$. All these indicate that we can free ourselves from any topological structure of $X$.

Therefore we adopt the following new assumptions on $A$ and $X$.

$\left(2.1^{\prime}\right)$ The space $X$ is a linear space and the operator $A_{B}$ is closeable in $X_{B}$. Denoting $\mathfrak{A}=\bar{A}_{B}^{X_{B}}$, we assume further that there exist subspaces $\mathfrak{X}$ satisfying $D(B) \subseteq \mathfrak{X} \subseteq X_{B}$, and $D_{B}(A) \subset D \subset \mathfrak{X} \cap D(\mathfrak{A})$ such that $\left(\lambda-\left.\mathfrak{A}\right|_{D}\right): D \rightarrow \mathfrak{X}$ is bijective for all $\lambda>\omega$.

We have then the following theorem [5].

THEOREM 2.6. Let the operators $A$ and $B$ satisfy the conditions of Definition 2.1 with assumption (2.2) replaced by assumption (2.1'). Then $A \in B-\mathscr{G}(M, \omega, X, Z)$ and (2.8) holds if and only if the following conditions are satisfied:

(1) $B(D)$ is dense in $Z_{B}$,

(2) there exist $M>0$ and $\omega \in \mathbb{R}$ such that for any $\mathfrak{y} \in \mathfrak{X}, \lambda>\omega$, and $n \in \mathbb{N}$ :

$$
\left\|\mathfrak{B}\left(\lambda I-\left.\mathfrak{A}\right|_{D}\right)^{-n} \mathfrak{y}\right\|_{Z} \leq \frac{M}{(\lambda-\omega)^{n}}\|\mathfrak{B} \mathfrak{y}\|_{Z}
$$

If we do not assume (2.8), then assumption (1) is sufficient but not necessary. In both cases the B-bounded semigroup is given again by (2.7).

Example 2.7. Consider $X=L_{2}\left(\mathbb{R}, e^{x^{2}} d x\right), A u=\partial_{x} u$ on the maximal domain, and $(B u)(x)=e^{-x^{2} / 2} u(x)$. Clearly, $B: X \rightarrow X$ is a continuous operator. Moreover, $\|B u\|_{X}=\|u\|_{L_{2}(\mathbb{R})}$ and since $C_{0}^{\infty}(\mathbb{R}) \subset X$, we can identify $X_{B}$ with $L_{2}(\mathbb{R})$. We consider the closure $\mathfrak{A}$ of $A$, that is, we take a sequence $\left(u_{n}\right)_{n \in \mathbb{N}}$ of elements of $D(A)$ such that $u_{n} \rightarrow u$ and $\partial_{x} u_{n} \rightarrow g$ as $n \rightarrow \infty$ in $L_{2}(\mathbb{R})$. However, this is the same as the closure of $D(A)$ in $W_{2}^{1}(\mathbb{R})$, and as $C_{0}^{\infty}(\mathbb{R}) \subset D(A)$ is dense in $W_{2}^{1}(\mathbb{R})$, we obtain that $\mathfrak{A} u=\partial_{x} u$ for $u \in W_{2}^{1}(\mathbb{R})$. Thus, $\mathfrak{A}$ generates a semigroup of contractions in $X_{B}$ and therefore $(Y(t) u)(x)=e^{-x^{2} / 2} u(t+x)$ satisfies conditions (1)-(3) of Definition 2.1. By 
standard argument (see also [5]) one can prove however that, $\lambda I-A: D(A) \rightarrow X$ is not bijective for any $\lambda$, hence Definition 2.1 is not applicable.

2.2. Special case. It is of interest to determine conditions under which $X_{B}$ is not an abstract space but can be identified with a subspace of $X$. We have the following theorem.

Theorem 2.8. Let $X, Z$ be Banach spaces and $B: X \rightarrow Z$ be an injective operator. The following conditions are equivalent:

(i) $X_{B}$ has the following properties:

$\left.\mathrm{i}^{\prime}\right)$ each coset $\tilde{x} \in X_{B}$ contains a sequence $\left(x_{n}\right)_{n \in \mathbb{N}}$ converging in the norm of $X$ to some $x \in X$, and $x$ is the limit of any other $X$-Cauchy sequence in $\tilde{x}$,

$\left(\mathrm{i}^{\prime \prime}\right)$ if $\left(x_{n}\right)_{n \in \mathbb{N}} \in \tilde{x},\left(y_{n}\right)_{n \in \mathbb{N}}$ satisfy $\left\|x_{n}-y_{n}\right\|_{X} \rightarrow 0$ as $n \rightarrow \infty$ and $\left(y_{n}\right)_{n \in \mathbb{N}} \in \tilde{y}$ for some $\tilde{y} \in X_{B}$, then $\tilde{x}=\tilde{y}$,

(ii) the operator $B$ is closeable and $B^{-1}$ is bounded,

(iii) there is an isometric isomorphism $T: X_{B} \rightarrow X_{B}^{\prime} \hookrightarrow X$ which satisfies $\left.T\right|_{D(B)}=\mathrm{Id}$.

Proof. (i) $\Leftrightarrow$ (ii). Since for each $\tilde{x} \in X_{B}$, there is exactly one representative $\left(x_{n}\right)_{n \in \mathbb{N}}$ which converges in $X$ to, say, $x$, the formula

$$
T \tilde{x}=T\left[\left(x_{n}\right)_{n \in \mathbb{N}}\right]=\lim _{n \rightarrow \infty} x_{n}=x
$$

defines an operator $T: X_{B} \rightarrow X$. Moreover, as $D(B) \ni \tilde{x}=[(x, x, \ldots)]$, we get $\left.T\right|_{D(B)}=$ Id. Next define

$X_{B}^{\prime}=\left\{x \in X ; x=\lim _{n \rightarrow \infty} x_{n}\right.$ for some $\left(x_{n}\right)_{n \in \mathbb{N}} \subset D(B)$ such that $\left(B x_{n}\right)_{n \in \mathbb{N}}$ is Cauchy $\}$.

By the assumption in $\left(\mathrm{i}^{\prime \prime}\right), T: X_{B} \rightarrow X_{B}^{\prime}$ is a bijection. This allows us to induce a norm in $X_{B}^{\prime}$ by

$$
\|x\|_{X_{B}^{\prime}}=\left\|\left[\left(x_{n}\right)_{n \in \mathbb{N}}\right]\right\|_{B}=\lim _{n \rightarrow \infty}\left\|B x_{n}\right\|,
$$

where $x=\lim _{n \rightarrow \infty} x_{n}$. In fact, if $x \in X_{B}^{\prime}$, then there must be $\tilde{x} \in X_{B}$ such that for some $\left(x_{n}\right)_{n \in \mathbb{N}} \in \tilde{x}, \lim _{n \rightarrow \infty} x_{n}=x$. By the assumption, there is only one such $\tilde{x}$, hence $x \in X_{B}^{\prime}$ determines uniquely $\tilde{x}$. Since $\left\|\left[\left(x_{n}\right)_{n \in \mathbb{N}}\right]\right\|_{B}$ is well defined by the last equality in (2.14), $\|\cdot\|_{X_{B}^{\prime}}$ is well defined. This norm turns $T$ into an isometry and thus $X_{B}^{\prime}$ becomes a Banach space.

Thanks to the assumption (i') again, we can define the shift $\mathfrak{B}^{\prime}: X_{B}^{\prime} \rightarrow Z$ by

$$
\mathfrak{B}^{\prime} x=\mathfrak{B} \tilde{x}=\lim _{n \rightarrow \infty} B x_{n},
$$

where $\left(x_{n}\right)_{n \in \mathbb{N}}$ is as before. We have

$$
\begin{gathered}
B: D(B) \stackrel{\text { onto }}{\longrightarrow} \operatorname{Im} B, \\
\mathfrak{B}^{\prime}: X_{B}^{\prime} \stackrel{\text { onto }}{\longrightarrow} \overline{\operatorname{Im} B} .
\end{gathered}
$$


Since $X_{B}^{\prime} \subset X$, we can consider $\left(\mathfrak{B}^{\prime}, X_{B}^{\prime}\right)$ as an unbounded operator in $X$. Let $\left(x_{n}\right)_{n \in \mathbb{N}} \subset$ $X_{B}^{\prime}$ converges to $x \in X$ and $\left(\mathfrak{B}^{\prime} x_{n}\right)_{n \in \mathbb{N}} \subset Z$ converges to $y \in Z$, in the respective norms. From the assumption (i'), for each $n \in \mathbb{N}$ there is a sequence $\left(x^{(n)}{ }_{k}\right)_{k \in \mathbb{N}} \in T^{-1} x_{n}$, $x_{k}^{(n)} \in D(B)$, converging to $x_{n}$ in $X$. From the construction of cosets in $X_{B},\left(B x_{k}^{(n)}\right)_{k \in \mathbb{N}}$ is a Cauchy sequence and as a consequence, for any $n \in \mathbb{N}, B x_{k}^{(n)} \rightarrow \mathfrak{B}^{\prime} x_{n}$ as $k \rightarrow \infty$. Indeed, denote $\tilde{x}_{n}=T^{-1} x_{n}=\left[\left(x_{k}^{(n)}\right)_{k \in \mathbb{N}}\right]$ and $\tilde{x}_{l}^{(n)}=T^{-1} x_{l}^{(n)}=\left[\left(x_{l}^{(n)}, x_{l}^{(n)}, \ldots\right)\right]$. Since $\left(B x_{k}^{(n)}\right)_{k \in \mathbb{N}}$ is a Cauchy sequence, we obtain, for any sufficiently large $l \in \mathbb{N}$

$$
\left\|\tilde{x}_{n}-\tilde{x}_{l}^{(n)}\right\|_{X_{B}}=\lim _{k \rightarrow \infty}\left\|B x_{k}^{(n)}-B x_{l}^{(n)}\right\|_{Z}<\epsilon,
$$

that is, $\lim _{l \rightarrow \infty} \tilde{x}_{l}^{(n)}=\tilde{x}_{n}$ in $X_{B}$. Since $T$ is an isomorphism, and by (2.14) and (2.15) we obtain that for any $n \in \mathbb{N}$,

$$
\lim _{k \rightarrow \infty} B x_{k}^{(n)}=\mathfrak{B}^{\prime} x_{n}
$$

For any $r \in \mathbb{N}$ we can find $n_{r} \in \mathbb{N}$ such that

$$
\left\|x_{n_{r}}-x\right\|_{X}<\frac{1}{r}, \quad\left\|\mathfrak{B}^{\prime} x_{n_{r}}-y\right\|_{Z}<\frac{1}{r},
$$

and for such a fixed $n_{r}$ we select $k_{r} \in \mathbb{N}$ satisfying

$$
\left\|x_{k_{r}}^{\left(n_{r}\right)}-x_{n_{r}}\right\|_{X}<\frac{1}{r}, \quad\left\|B x_{k_{r}}^{\left(n_{r}\right)}-\mathfrak{B} x_{n_{r}}\right\|_{Z}<\frac{1}{r} .
$$

Define $\phi_{r}=x_{k_{r}}^{\left(n_{r}\right)}$. For any $r \in \mathbb{N}, \phi_{r} \in D(B)$. Fixing $\epsilon>0$ we find $r_{0}>2 / \epsilon$, and for any $r>r_{0}$ we have by estimates (2.19) and (2.20),

$$
\begin{gathered}
\left\|\phi_{r}-x\right\|_{X}<\frac{2}{r}<\epsilon, \\
\left\|B \phi_{r}-y\right\|_{Z}=\left\|B \phi_{r}-\mathfrak{B}^{\prime} \bar{x}\right\|_{Z}<\frac{2}{r}<\epsilon,
\end{gathered}
$$

which means that $\left(\phi_{r}\right)_{r \in \mathbb{N}}$ is a sequence defining $\bar{x}$ and from assumption (i') we obtain $\bar{x}=x \in X_{B}^{\prime}$. Thus $\left(\mathfrak{B}^{\prime}, X_{B}^{\prime}\right)$ is a closed operator in $X$. Therefore $X_{B}^{\prime}=D\left(\mathfrak{B}^{\prime}\right)$, equipped with the norm of the graph, is a Banach space, and $\mathfrak{B}^{\prime}: X_{B}^{\prime} \stackrel{\text { onto }}{\longrightarrow} \overline{\operatorname{Im} B}$ is a continuous bijection. Therefore the inverse of $\mathfrak{B}^{\prime}$ is bounded in the norm of the graph, and for its restriction $B^{-1}$ we obtain

$$
\forall y \in \operatorname{Im} B,\|x\|_{X} \leq\|x\|_{X}+\|B x\|_{Z}=\|x\|_{D\left(\mathfrak{B}^{\prime}\right)} \leq K\|B x\|_{Z}
$$

for some constant $K$, which gives the boundedness of $B^{-1}: Z \rightarrow X$.

Now let $B: X \rightarrow Z$ be a closeable operator with a bounded inverse. For any $\tilde{x}$, let $\left(x_{n}\right)_{n \in \mathbb{N}} \in \tilde{x}$, that is, $\left(B x_{n}\right)_{n \in \mathbb{N}}$ is a Cauchy sequence in $Z$. By the boundedness of $B^{-1}$, $\left(x_{n}\right)_{n \in \mathbb{N}}$ is a Cauchy sequence in $X$ and its limit $x \in D(\bar{B})$. Also, by the boundedness of $B^{-1}$, if any other Cauchy sequence $\left(B y_{n}\right)_{n \in \mathbb{N}}$ is such that $\left(B\left(x_{n}-y_{n}\right)\right)_{n \in \mathbb{N}}$ is convergent to 0 , then $\lim _{n \rightarrow \infty} y_{n}=x$. Next, if $x_{n} \rightarrow x$ and $y_{n} \rightarrow x$ in $X$ determine two cosets $\tilde{x}$ and $\tilde{y}$, then by definition both $\left(B x_{n}\right)_{n \in \mathbb{N}}$ and $\left(B y_{n}\right)_{n \in \mathbb{N}}$ converge. Since $B$ is closeable, they must have the same limit, that is, $\tilde{x}=\tilde{y}$. 
(ii) $\Leftrightarrow$ (iii). From (ii) to (iii) the theorem follows easily, as in the previous part we constructed a required isometry, and since $X_{B}^{\prime}=D(\bar{B})$, set-theoretically and topologically, $X_{B}^{\prime} \hookrightarrow X$.

Conversely, let $T$ be the stipulated isomorphism and define for $x \in X_{B}^{\prime}$

$$
\mathbb{B} x=\mathfrak{B} T^{-1} x \text {. }
$$

Since $X_{B}^{\prime} \hookrightarrow X$, then $T: X_{B} \rightarrow X$ is continuous, and therefore $T^{-1}$ is closed as an operator defined in $X$. Since $\mathfrak{B}$ is an isomorphism, then $\mathbb{B}: X \rightarrow \overline{\operatorname{Im} B}$ is closed. But since $\left.T\right|_{D(B)}=\mathrm{Id}, \mathbb{B}$ is an extension of $B$ and therefore $B$ is closeable. Moreover, $\mathbb{B}^{-1}=T \mathfrak{B}^{-1}: \overline{\operatorname{Im} B} \rightarrow X$ is continuous, therefore $B^{-1}=\left.T \mathfrak{B}^{-1}\right|_{\operatorname{Im} B}$ is also bounded.

Remark 2.9. It is interesting to note that there exist operators which are injective with bounded inverse but which are not closeable [17].

If $B$ is not invertible, then we can still obtain a similar result, however in a less compact form. First we note that it is impossible to follow the way leading to Theorem 2.3 as already the quotient space $D(B) / N(B)$ is not a subspace of $X$.

We start with some preliminaries. Following, for example, [21, pages 766-767], we can write split $D(B)$ as an algebraic direct sum

$$
D(B)=N(B) \oplus D_{B}
$$

for some linear space $D_{B}$ such that $B: D_{B} \rightarrow R(B)$ is a bijection (clearly $D_{B}$ is not unique, but $R(B)$ is independent of the choice of $D_{B}$ ). We denote by $B_{R}^{-1}: R(B) \rightarrow D_{B}$ the right inverse to $B$ having $D_{B}$ as its range. Conversely, given any right inverse $B_{R}^{-1}$ to $B$ defined on $R(B)$ we can always split $D(B)$ as in (2.24) with $D_{B}=R\left(B_{R}^{-1}\right)$.

Now we can construct a number of extrapolation spaces $\tilde{X}_{B}$ completing various $D_{B}$ 's in the norm $\|B \cdot\|_{Z}$. It is important to note that all these spaces are isometrically isomorphic to each other and also to the space $X_{B}$. Indeed, let $\tilde{X}_{B}$ be a completion of $D_{B}$ with respect to the abovementioned norm. Denote by $\tilde{\mathfrak{B}}: \tilde{X}_{B} \rightarrow \overline{R(B)}^{Z}$ the extension by continuity of $\left(B, D_{B}\right)$. As in the original case, this is an isometric isomorphism. However, also $\mathfrak{B}: X_{B} \rightarrow \overline{R(B)}^{Z}$ is an isometric isomorphism, therefore $\mathfrak{T}=\tilde{\mathfrak{B}}^{-1} \mathfrak{B}$ : $X_{B} \rightarrow \tilde{X}_{B}$ is an isometric isomorphism. Let $x \in D_{B} \subset D(B)$, then $\mathfrak{B} x=B x \in R(B)$, but $\tilde{B}^{-1}$ is here the extension of the inverse of $\left.B\right|_{D_{B}}$, hence we obtain

$$
\left.\mathfrak{T}\right|_{D_{B}}=\mathrm{Id}
$$

Now we can formulate a generalization of Theorem 2.8.

Corollary 2.10. Let $X, Z$ be Banach spaces, $X=N(B) \oplus D_{B}$ and $D_{B}$ be the range of some right inverse $B_{R}^{-1}$. The following are equivalent:

(i) the operator $\left(B, D_{B}\right)$ is closeable and $B_{R}^{-1}$ is bounded,

(ii) there is an isomorphism $T: X_{B} \rightarrow X_{B}^{\prime} \hookrightarrow X$ which satisfies $\left.T\right|_{D_{B}}=\mathrm{Id}$. 
If either of the above condition holds and additionally both $N(B)$ and $D_{B}$ are closed, then $(B, D(B))$ is also closeable.

Proof. (i) $\Leftrightarrow$ (ii). The proof is straightforward by repeating the proof of the equivalence (ii) $\Leftrightarrow$ (iii) of Theorem 2.8 with $(B, D(B))$ replaced by $\left(B, D_{B}\right)$ and applying (2.25).

To prove the last statement consider $x_{n} \rightarrow 0$ in $X, x_{n} \in D(B), n=1, \ldots$, such that $B x_{n} \rightarrow y$ in $Z$. We write $x_{n}=x_{n}^{\prime}+x_{n}^{\prime \prime}$ with $x_{n}^{\prime} \in N(B)$ and $x_{n}^{\prime \prime} \in D_{B}$ for $n=1, \ldots$ Since $B x_{n}=B x_{n}^{\prime \prime}$, we see that $\left(B x^{\prime \prime}{ }_{n}\right)_{n \in \mathbb{N}}$ is also convergent, and by the boundedness of the right inverse, $\left(x^{\prime \prime}{ }_{n}\right)_{n \in \mathbb{N}}$ is also convergent in $X$. Therefore $\left(x^{\prime}{ }_{n}\right)_{n \in \mathbb{N}}$ converges and by the closedness of both subspaces we get $x^{\prime}=\lim _{n \rightarrow \infty} x_{n}^{\prime} \in N(B)$ and $x^{\prime \prime}=$ $\lim _{n \rightarrow \infty} x_{n}^{\prime \prime} \in D_{B}$. On the other hand, $x^{\prime}+x^{\prime \prime}=0$ which yields $x^{\prime}=x^{\prime \prime}=0$ by (2.24). Thus $x_{n}^{\prime \prime} \rightarrow 0$ and $B x_{n}^{\prime \prime} \rightarrow y$ implies $y=0$ by closeability of $\left(B, D_{B}\right)$ and therefore $(B, D(B))$ is also closed.

If we have the case described in Theorem 2.8 , the operator $\mathfrak{A}$ also becomes much simpler.

THEOREM 2.11. If $B$ is a closeable operator such that $B^{-1}$ is bounded, and $A$ is closeable in $X$ with $\lambda I-\bar{A}$ injective for some $\lambda>\omega$, and moreover $A \in B-$ $\mathscr{G}(M, \omega, X, Z)$, then

$$
\mathfrak{A}=\left.\bar{A}\right|_{D_{\bar{B}}(\bar{A})},
$$

where

$$
D_{\bar{B}}(\bar{A})=\{x \in D(\bar{A}) \cap D(\bar{B}) ; \bar{A} x \in D(\bar{B})\} .
$$

Proof. Let $x \in D(\mathfrak{A})$. Then there exists a sequence $\left(x_{n}\right)_{n \in \mathbb{N}}$ in $D_{B}(A)$ such that $B x_{n} \rightarrow$ $\bar{B} x$ and $B A x_{n} \rightarrow \bar{B} \mathfrak{A} x$ as $n \rightarrow \infty$. From the proof of Theorem 2.8, it follows that also $\bar{B}^{-1}$ is bounded, that is, $x_{n} \rightarrow x$ and $A x_{n} \rightarrow \mathfrak{A} x$ in $X$ as $n \rightarrow \infty$. From this formulae we get that $x \in D(\bar{B}), x \in D(\bar{A})$ with $\mathfrak{A} x=\bar{A} x$ and $\bar{A} x \in D(\bar{B})$, that is, $D(\mathfrak{A}) \subset D_{\bar{B}}(\bar{A})$.

Conversely, for $\lambda>\omega, \lambda I-\mathfrak{A}: D(\mathfrak{A}) \stackrel{\text { onto }}{\longrightarrow} D(\bar{B})$, and for $x \in D_{\bar{B}}(\bar{A}), \lambda x-\bar{A} x \in$ $D(\bar{B})$. Thus for some $y \in D(\mathfrak{A})$ we obtain

$$
\lambda y-\mathfrak{A} y=\lambda x-\bar{A} x,
$$

but $y \in D(\mathfrak{A}) \subset D_{\bar{B}}(\bar{A})$ yields $\mathfrak{A} y=\bar{A} y$, and by injectivity of $\lambda I-\bar{A}$ we get $x=y \in D(\mathfrak{A})$.

Example 2.12. We consider $X=L_{2}\left(\mathbb{R}, e^{-x^{2}} d x\right), A u=\partial_{x} u$ on the maximal domain, and $(B u)(x)=e^{x^{2} / 2} u(x) . B: X \rightarrow X$ is an unbounded operator and since $\|B u\|_{X}=$ $\|u\|_{L_{2}(\mathbb{R})}$, we see that $D(B)=L_{2}(\mathbb{R})$. Since $B(D(B))=X$, we obtain that $X_{B}=$ $D(B)=L_{2}(\mathbb{R})$ by Theorem 2.8. Then $D_{B}(A)=W_{2}^{1}(\mathbb{R})$ and $A$ generates a contraction semigroup, say $(T(t))_{t \geq 0}$, in $L_{2}(\mathbb{R})$. Thus $Y(t) u=B T(t) u=e^{x^{2} / 2} u(t+x)$ is the $B$-bounded semigroup generated by $(A, B)$.

Note that here neither $D(A) \subset D(B)$, nor $\rho(A) \supset[\omega, \infty$ [, but the (2.5) is satisfied. 
$B$-bounded semigroups and implicit evolution equations

\section{3. $B$-bounded semigroups and implicit evolution equations}

3.1. $X_{B}$-solutions of implicit evolution equations. We consider again the original Cauchy problem (1.1). It is often the case that the original spaces $X$ and $Z$ are not the most convenient spaces from the mathematical point of view. We are usually interested to keep the values of the solution in the original space which may be related to some physical properties like finite total energy space, finite mass, and so forth, but for (1.1) to hold in the strict sense may be too restrictive and often it is enough that it holds in some other Banach (or even linear topological) space $\tilde{X}$ with $K$ and $L$ replaced by appropriate extensions $\tilde{K}$ and $\tilde{L}$ acting from $Z$ to $\tilde{X}$. This concept is similar to the differentiation in the sense of distributions; a related concept for semigroups is sometimes called the Haraux extrapolation [13].

To be able to link $K$ and $L$ with $\tilde{K}$ and $\tilde{L}$ we restrict these extensions to the closures of respective operators. In other words, $D(L)$ and $D(K)$ are required to be cores for $\tilde{L}$ and $\tilde{K}$, respectively.

As we mentioned in the introduction, in general, thanks to Theorem 2.6, we do not need any topological structure in $X$ and therefore there is no need to introduce any topological assumptions on $K$ and $L$ separately - as we see, these will be replaced by appropriate assumption imposed on either $L K^{-1}$ or $K^{-1} L$.

We introduce the following definition.

Definition 3.1. Let $X \subset \tilde{X}$ and $\tilde{L}=\bar{L}^{\tilde{X}}, \tilde{K}=\bar{K}^{\tilde{X}}$. A $Z$-valued function $t \rightarrow u(t)$ is called an $\tilde{X}$-solution of the problem (1.1) if it is a classical solution of the problem

$$
\frac{d}{d t}(\tilde{K} u)=\tilde{L} u, \quad \lim _{t \rightarrow 0^{+}}(\tilde{K} u)(t)=\stackrel{\circ}{u}
$$

that is, $t \rightarrow \tilde{K} u(t)$ is continuously differentiable in $\tilde{X}$, the differential equation holds for all $t>0$ in $\tilde{X}$, and the initial condition holds as a limit in the topology of $\tilde{X}$.

With this definition we can formulate the following theorem.

Theorem 3.2. Suppose that we are given operators $K: D(K) \rightarrow X$ and $L: D(L) \rightarrow X$ with $D(L), D(K) \subset Z$, where $Z$ is a Banach space and $X$ is a linear space. Assume that $K$ is a densely defined, one-to-one operator. Define $A=L K^{-1}$ with the natural domain $D(A)=K(D(L) \cap D(K))$ and $B=K^{-1}$. If $A \in B-\mathscr{G}(M, \omega, X, Z)$ (in the sense of Subsection 2.1), then, for any $x \in D_{B}(A)=\left\{x \in K(D(L) \cap D(K)) ; L K^{-1} x \in \operatorname{Im} K\right\}$, the function $t \rightarrow Y(t) x$, where $(Y(t))_{t \geq 0}$ is the B-bounded semigroup generated by $A$, is an $X_{B}$-solution of the problem (1.1).

Proof. Since $Y(t) x=\mathfrak{B} \exp (t \mathfrak{A}) x$, where $\mathfrak{B}: X_{B} \rightarrow Z$ is an isomorphism and $x \in$ $D(\mathfrak{A})$, we obtain

$$
\frac{d}{d t} Y(t) x=\frac{d}{d t} \mathfrak{B} \exp (t \mathfrak{A}) \mathfrak{p} x=\mathfrak{B} \frac{d}{d t} \exp (t \mathfrak{A}) \mathfrak{p} x=\mathfrak{B A} \exp (t \mathfrak{A}) \mathfrak{p} x
$$


which can be rewritten as

$$
\frac{d}{d t} \mathfrak{B}^{-1} Y(t) x=\mathfrak{A}^{-1} Y(t) x
$$

Similarly,

$$
\lim _{t \rightarrow 0^{+}} \mathfrak{B}^{-1} Y(t) x=x .
$$

To complete the proof we must show that $\mathfrak{B}^{-1}$ is the closure of $K=B^{-1}$ and that $\mathfrak{A}^{-1}$ is the closure of $L=A B^{-1}$. The first statement follows from the fact that $\mathfrak{B}$, being the extension by density, is also the closure of $B$ and that the operation $(x, y) \rightarrow(y, x)$, which transforms graph of an operator onto the graph of its inverse, is an isomorphism.

Consider the second operator. By construction $\mathfrak{A}$ is the closure of $L K^{-1}$ defined on

$$
D_{B}(A)=D\left(L K^{-1}\right)=\left\{x \in K(D(L) \cap D(K)) ; L K^{-1} x \in \operatorname{Im} K\right\}
$$

in $X_{B}$.

We know that $\mathfrak{B}$ is the closure of $K^{-1}$ in $X_{B}$. Consider the operator $\left(\mathfrak{A} \mathfrak{B}^{-1}, \mathfrak{B} D(\mathfrak{A})\right)$; it is well defined and closed, as $\mathfrak{A}$ is closed and $\mathfrak{B}^{-1}$ is continuous. Also, since $D(\mathfrak{A})$ is dense in $X_{B}$ and $\mathfrak{B}$ is an isomorphism, this is a densely defined operator.

We prove that the operator $\left(\mathfrak{A B}^{-1}, \mathfrak{B} D(\mathfrak{A})\right)$ is the closure of $(L, D(L) \cap D(K))$ in $X_{B}$. Let $x \in D(L) \cap D(K)$, then $K x \in K(D(L) \cap D(K)) \subset D(A)$. For such $x$ we have

$$
\mathfrak{A B}^{-1} x=\mathfrak{A} K x=L K^{-1} K x=L x,
$$

hence $\mathfrak{A P}^{-1}$ is an extension of $(L, D(L))$.

We know that $\mathfrak{A}$ is the $X_{B}$-closure of $L K^{-1}$ from $D_{B}(A)$, that is for any $y \in D(\mathfrak{A})$ there is a sequence $\left(y_{n}\right)_{n \in \mathbb{N}}$ of elements belonging to $K(D(L) \cap D(K))$ and such that $L K^{-1} y_{n} \in \operatorname{Im} K$, which satisfies $y_{n} \rightarrow y$ in $X_{B}$ and

$$
\mathfrak{A} y=X_{B}-\lim _{n \rightarrow \infty} L K^{-1} y_{n} .
$$

Take arbitrary $x \in \mathfrak{B}(D(\mathfrak{A}))$; then $x=\mathfrak{B} y$ for some $y \in D(\mathfrak{A})$ or $y=\mathfrak{B}^{-1} x$. Now

$$
\mathfrak{A} y=\mathfrak{A} \mathfrak{B}^{-1} x=X_{B}-\lim _{n \rightarrow \infty} L K^{-1} y_{n},
$$

but $y_{n} \in K(D(L) \cap D(K))$, that is, $y_{n}=K x_{n}$ for some $x_{n} \in D(L) \cap D(K)$. However, on $D(K)=\operatorname{Im} B$ we have $\mathfrak{B}^{-1} x=B^{-1} x=K x$ and consequently,

$$
y_{n}=K x_{n}=\mathfrak{B}^{-1} x_{n} .
$$

Because $\left(y_{n}\right)_{n \in \mathbb{N}}$ converges in $X_{B}$ by construction, $\left(x_{n}\right)_{n \in \mathbb{N}}=\left(\mathfrak{B} y_{n}\right)_{n \in \mathbb{N}}$ converges in $Z$ to $x$ (by continuity of $\mathfrak{B}$ ) and

$$
\mathfrak{A} \mathfrak{B}^{-1} x=\mathfrak{A} y=X_{B}-\lim _{n \rightarrow \infty} L K^{-1} y_{n}=X_{B}-\lim _{n \rightarrow \infty} L x_{n} .
$$

This shows that $\mathfrak{A B}^{-1} \subset{\overline{L K^{-1}}}^{X}$. However, since $\mathfrak{A B}^{-1}$ is a closed extension of $L K^{-1}$, the theorem is proved. 
Remark 3.3. The assumption that $K$ is densely defined in $Z$ is not essential. If it does not hold, then in the considerations the space $Z$ should be replaced by $\overline{D(K)}{ }^{Z}=Z_{B}$.

The assumption (2.2), specified to the present conditions, means that the operator $\left(L K^{-1}, D\left(L K^{-1}\right)\right)$, where $D\left(L K^{-1}\right)$ is defined by (3.5), satisfies

$$
\rho\left(L K^{-1}\right) \supset[\omega, \infty[
$$

for some $\omega \in \mathbb{R}$. If this assumption is satisfied, then we can combine Theorem 3.2 with Corollary 2.5 to obtain the following result.

\section{THeorem 3.4. Assume that}

(1) the set $\{y \in D(L) \cap D(K) ; L y \in \operatorname{Im} K\}$ is dense in $\overline{D(K)}^{Z}$,

(2) for $x \in D(K)$ either

$$
\left\|\left(\lambda I-K^{-1} L\right)^{-1} x\right\|_{Z} \leq \lambda^{-1}\|x\|_{Z}, \quad \lambda>0,
$$

or

$$
\left\|\left(\lambda I-K^{-1} L\right)^{-1} x\right\|_{Z} \leq \frac{M}{|\lambda-\omega|}\|x\|_{Z}, \quad \lambda \in S_{\theta},
$$

then, for any $x \in\left\{x \in K(D(L) \cap D(K)) ; L K^{-1} x \in \operatorname{Im} K\right\}$, the function $t \rightarrow$ $Y(t) x$ is an $X_{B}$-solution to (1.1).

In reflexive spaces assumption (1) is superfluous.

Proof. Assumption (1) of Theorem 2.4 requires $B\left[D_{B}(A)\right]$ to be dense in $Z_{B}$. With $B=K^{-1}$ and $D_{B}(A)$ defined by (3.5), we obtain

$$
\begin{aligned}
B\left[D_{B}(A)\right] & =K^{-1}\left\{x \in K(D(L) \cap D(K)) ; L K^{-1} x \in \operatorname{Im} K\right\} \\
& =\{y \in D(L) \cap D(K) ; L y \in \operatorname{Im} K\}
\end{aligned}
$$

so our assumption (1) is simply rephrasing of that of Theorem 2.4.

Equation (2.9) specified to our case reads

$$
\left\|K^{-1}\left(\lambda I-L K^{-1}\right)^{-1} f\right\|_{Z} \leq \lambda^{-1}\left\|K^{-1} f\right\|_{Z}, \quad \lambda>0,
$$

for $f \in \operatorname{Im} K$. Since $K$ is invertible, putting $x=K^{-1} f$ gives an arbitrary element of $D(K)$. Hence we have

$$
\begin{aligned}
\left\|K^{-1}\left(\lambda I-L K^{-1}\right)^{-1} K x\right\|_{Z} & =\left\|\left(K^{-1}\left(\lambda I-L K^{-1}\right) K\right)^{-1} x\right\|_{Z} \\
& =\left\|\left(\lambda I-K^{-1} L\right)^{-1} x\right\|_{Z},
\end{aligned}
$$

thus inequality (3.15) follows from (3.12). Analogous arguments prove the statement for analytic case. Assumption (1) is needed for the density of the domain of $\mathfrak{A}$ in $X_{B}$, and it is well known, that for reflexive space this follows once the Hille-Yosida estimates are satisfied. 
An important role in the theory of $B$-bounded semigroups is played by the operator $B A B^{-1}$ which gives rise to another semigroup which can be used to define the $B$ bounded semigroup, the advantage of which stems from acting in the space $Z$ rather than in the abstract space $X_{B}$. In our case formally we have $B A B^{-1}=K^{-1} L K^{-1} K=$ $K^{-1} L$. Precisely, by (3.5) we must define $K^{-1} L$ on the domain

$$
D\left(K^{-1} L\right)=\{y \in D(L) \cap D(K) ; L y \in \operatorname{Im} K\},
$$

which has already appeared in Theorem 3.4. Then we have the following proposition.

Proposition 3.5. The operator $\left(L K^{-1}, D\left(L K^{-1}\right)\right)$ is closeable in $X_{B}$ if and only if $\left(K^{-1} L, D\left(K^{-1} L\right)\right)$ is closeable in $Z$ and the following equality holds:

$$
\mathfrak{A} x=\mathfrak{K} \bar{K}^{-1} L \mathfrak{K}^{-1} x,
$$

where $\mathfrak{K}$ is the $X_{B}$-closure of $K$. Consequently, the operator $\mathfrak{B A}^{-1}$ of Theorem 2.3 is equal to $\overline{K^{-1} L}$.

Proof. Let $x \in D(\mathfrak{A})$, then

$$
y=\mathfrak{K}^{-1} x=\lim _{n \rightarrow \infty} K^{-1} x_{n}=\lim _{n \rightarrow \infty} y_{n}
$$

in $Z$, with $x_{n} \in D_{B}(A)$, hence $y_{n} \in D\left(K^{-1} L\right)$, and

$$
\mathfrak{K}^{-1} \mathfrak{A} x=\lim _{n \rightarrow \infty} K^{-1} L K^{-1} x_{n}=\lim _{n \rightarrow \infty} K^{-1} L y_{n} .
$$

This shows that $y \in D\left(\overline{K^{-1} L}\right)$ and $\overline{K^{-1} L} y=\mathfrak{K}^{-1} \mathfrak{A} x$ and consequently

$$
\mathfrak{A} \subset \mathfrak{K} \overline{K^{-1} L} \mathfrak{K}^{-1} .
$$

Conversely, if $y \in D\left(\overline{K^{-1} L}\right)$, then for $\left(y_{n}\right)_{n \in \mathbb{N}} \subset D\left(K^{-1} L\right)$ we have

$$
\lim _{n \rightarrow \infty} y_{n}=y, \quad \lim _{n \rightarrow \infty} K^{-1} L y_{n}=\overline{K^{-1} L} y .
$$

Putting $x_{n}=K y_{n}$, we obtain that $\left(x_{n}\right)_{n \in \mathbb{N}}$ converges in $X_{B}$ and by continuity of $\mathfrak{K}$, we have $X_{B}-\lim _{n \rightarrow \infty} x_{n}=x=\mathfrak{K} y$. Thus, $\left(K^{-1} L K^{-1} x_{n}\right)_{n \in \mathbb{N}}$ converges in $Z$, and therefore $\left(L K^{-1} x_{n}\right)_{n \in \mathbb{N}}$ converges in $X_{B}$ to $\mathfrak{A} x$ and $\mathfrak{K} \overline{K^{-1} L} \mathfrak{K}^{-1} \subset \mathfrak{A}$.

With this proposition, Theorem 2.6 yields a stronger version of Theorem 3.4. Note that the assumption $\left(2.1^{\prime}\right)$ is incorporated into Theorem 3.6 as assumption (2) below.

THEOREM 3.6. Assume that

(1) $D\left(K^{-1} L\right)$ is dense in $\overline{D(K)}^{Z}$,

(2) the operator $\left(K^{-1} L, D\left(K^{-1} L\right)\right)$ is closeable in $Z$ and there exist spaces: $\mathfrak{X}$ satisfying for $D(K) \subseteq \mathfrak{X} \subseteq Z$, and $D$ satisfying $D\left(K^{-1} L\right) \subseteq D \subseteq D\left(\overline{K^{-1} L}\right)$ such that for $x \in \mathscr{X}$ either

$$
\left\|\left(\lambda I-\left.\overline{K^{-1} L}\right|_{D}\right)^{-1} x\right\|_{Z} \leq \lambda^{-1}\|x\|_{Z}, \quad \lambda>0,
$$


or

$$
\left\|\left(\lambda I-\left.\overline{K^{-1} L}\right|_{D}\right)^{-1} x\right\|_{Z} \leq \frac{M}{|\lambda-\omega|}\|x\|_{Z}, \quad \lambda \in S_{\theta},
$$

then, for any $x \in D\left(L K^{-1}\right)$, the function $t \rightarrow Y(t) x$ is an $X_{B}$-solution to (1.1). For $x \in D\left(L K^{-1}\right)$ the classical solution is given by

$$
u(t, x)=e^{t \overline{K^{-1} L}} K^{-1} x .
$$

In reflexive spaces assumption (1) is superfluous.

Remark 3.7. An $X_{B}$-solution to (1.1) exists for a larger class of initial values, namely for all $x \in \mathfrak{K} D\left(\overline{K^{-1} L}\right)$.

Remark 3.8. Using arguments similar to that preceding Corollary 2.10, we can provide analogous solvability results even when $K$ is not invertible. In fact, all the theorems above are valid if we replace $(K, D(K))$ by $\left(K, D_{K}\right)$ where $Z=N(K) \oplus D_{K}$, and $Z$ by $Z_{B}=\bar{D}_{K}^{Z}$ as noted in Remark 3.3. However, in such a case the uniqueness is an open question, as different choices of $D_{K}$ lead in general to different spaces $X_{B}$, and to different $B$-bounded semigroups.

3.2. $B$-bounded semigroups and empathy. We start with a brief outline of the empathy theory as presented in [19]. Let $X$ and $Z$ be Banach spaces and consider two families of operators $\mathscr{E}=\{E(t): X \rightarrow X\}_{t>0}$ and $\mathscr{S}=\{S(t): X \rightarrow Z\}_{t>0}$ such that the Laplace transforms: $R(\lambda) y=\mathscr{L}(E(t) y)(\lambda)$ and $P(\lambda) y=\mathscr{L}(S(t) y)(\lambda)$ exist for any $y \in X$ and $\lambda>0$. The pair $\langle\mathscr{Y}, \mathscr{E}\rangle$ is called an empathy if for any $s, t>0$

$$
S(t+s)=S(t) E(s)
$$

and $P(\xi)$ is invertible for some $\xi>0$. It can be proved that then $P(\lambda)$ is invertible for any $\lambda>0$, and the same is valid for $R(\lambda)$. Also, the subspaces $\mathscr{D}_{E}=R(\lambda) X$ and $\mathscr{D}=$ $P(\lambda) Z$ are independent of $\lambda$. Define the operators: $K: \mathscr{D} \rightarrow \mathscr{D}_{E}$ by $K=R(\lambda) P^{-1}(\lambda)$ and $L: \mathscr{D} \rightarrow X$ by $L=(\lambda R(\lambda)-I) P^{-1}(\lambda)$; both can be proved to be independent of $\lambda$ and

$$
P(\lambda)=(\lambda K-L)^{-1}
$$

The pair $\langle L, K\rangle$ is called the generator of empathy $\langle\mathscr{Y}, \mathscr{E}\rangle$. In this case, for any $y \in \mathscr{D}_{E}$, the function $t \rightarrow S(t) y$ is a solution of the Cauchy problem (1.1). This result is not satisfactory, as it allows to recognize which Cauchy problem is solvable by a given empathy. The inverse requires an additional assumption. To explain its meaning, we first note that it can be proved that $\mathscr{E}$ is a semigroup, but not necessarily a $C_{0}$-semigroup, in $X$. In particular, the function $t \rightarrow E(t) x$ can be unbounded at $t=0$ for some $x \in X$. To be able to prove the main generation result for empathy in [19], the author introduced the assumption that the empathy $\langle\mathscr{Y}, \mathscr{E}\rangle$ is uniformly bounded, that is, there exist constants $N^{\prime}, M^{\prime}$ such that $\sup _{t>0}\|S(t)\| \leq M^{\prime}, \sup _{t>0}\|E(t)\| \leq N^{\prime}$. Then the following theorem is valid. 
THeOREM 3.9. Suppose that the space $X$ has the Radon-Nikodym property. The operator pair $\langle L, K\rangle$ is the generator of a uniformly bounded empathy $\langle\mathscr{Y}, \mathscr{E}\rangle$ if and only if the operators $P(\lambda)$ and $R(\lambda)$ are bounded for every $\lambda>0$ and there exist positive numbers $M, N$ such that for every $\lambda>0$ and $k=1,2, \ldots$

$$
\|P(\lambda)\| \leq \lambda^{-1} M, \quad\left\|R^{k}(\lambda)\right\| \leq \lambda^{-k} N
$$

To compare empathy with $B$-bounded semigroups, we first note that for $\langle L, K\rangle$ to be the generator of an empathy, $K$ must be an injective operator. Moreover, by definition, the solution family $(S(t))_{t \geq 0}$ is a family of bounded operators in $X$. Since the $B$ bounded semigroup $(Y(t))_{t \geq 0}$ is supposed to give solutions for the same problem, it must be also defined on the whole space, which requires $B=K^{-1}$ to be a bounded operator.

We have the following theorem.

TheOREM 3.10. Let $(Y(t))_{t \geq 0}$ be a B-bounded semigroup generated by $A$ where $A$ : $D(A) \rightarrow X, B: D(B) \rightarrow Z, D(A), D(B) \subset X, A$ satisfies (2.2) with $\omega=0$, and $B$ is a bounded, one-to-one operator. The pair $\left\langle(Y(t))_{t \geq 0},\left(\left.e^{t \mathfrak{A}}\right|_{X}\right)_{t \geq 0}\right\rangle$ is an empathy generated by $\left\langle A B^{-1}, B^{-1}\right\rangle$ if and only if

$$
\begin{gathered}
\forall t \geq 0, e^{t \mathfrak{A}} X \subset X \\
\forall x \in X, \lambda>0, t \longrightarrow e^{-\lambda t}\left(\left.e^{t \mathfrak{A}}\right|_{X}\right) x \in L_{1}(0, \infty, X) .
\end{gathered}
$$

Proof. Since $B$ is a bounded operator, $(Y(t))_{t \geq 0}$ is a family of bounded operators by property (1) of Definition 2.1. Moreover, by [8] we have

$$
P(\lambda) x=B(\lambda I-A)^{-1} x=\int_{0}^{\infty} e^{-\lambda t} Y(t) x d t
$$

for any $x \in X$ and since $B$ is invertible, $P(\lambda)$ exists and is invertible.

By (3.30) the operator $R(\lambda)$ is well defined. Fix $t \geq 0$ and consider $x_{n} \rightarrow x$ and $e^{t \mathfrak{A}} x_{n} \rightarrow y$ in $X$ as $n \rightarrow \infty$. Since by the boundedness of $B, X \hookrightarrow X X_{B}, y=e^{t \mathfrak{A}} x$ and $e^{t \mathfrak{A}}$ is a closed operator, and being defined on the whole $X$, it is a bounded operator. Next, for any $x \in X$ we have, by (3.29),

$$
Y(t+s) x=\mathfrak{B} \exp ((t+s) \mathfrak{A}) x=\mathfrak{B} \exp (t \mathfrak{A}) \exp (s \mathfrak{A}) x=Y(t) \exp (s \mathfrak{A}) x,
$$

therefore $\left\langle(Y(t))_{t \geq 0},\left(\left.e^{t \mathfrak{A}}\right|_{X}\right)_{t \geq 0}\right\rangle$ is an empathy.

Since $B$ is bounded, we obtain for any $x \in X$

$$
B R(\lambda) x=\left.B \int_{0}^{\infty} e^{-\lambda t} e^{t \mathfrak{A}}\right|_{X} x d t=\int_{0}^{\infty} e^{-\lambda t} Y(t) x d t=B(\lambda I-A)^{-1} x,
$$

where again we used $X \hookrightarrow X_{B}$ to obtain the equivalence of the integrals. Hence $R(\lambda)=$ $(\lambda I-A)^{-1}$, by a simple calculation

$$
\begin{gathered}
K=R(\lambda) P^{-1}(\lambda)=B^{-1}, \\
L=\left(\lambda(\lambda I-A)^{-1}-I\right)(\lambda I-A) B^{-1}=A B^{-1} .
\end{gathered}
$$


Conversely, the properties (3.29) and (3.30) follow from the original definition of empathy.

A better characterization of $\left(\left.e^{t \mathfrak{A}}\right|_{X}\right)_{t \geq 0}$ can be obtained when the empathy is uniformly bounded. Clearly, then the statement of Theorem 3.10 is valid provided conditions (3.29) and (3.30) are replaced by the requirement that $\left(\left.e^{t \mathfrak{A}}\right|_{X}\right)_{t \geq 0}$ is a strongly measurable and uniformly bounded semigroup in $X$. Depending on the structure of $X$, we can prove some additional properties of this semigroup.

Proposition 3.11. Let the assumptions of Theorem 3.10 be satisfied and $\left\langle(Y(t))_{t \geq 0}\right.$, $\left.\left(\left.e^{t \mathfrak{A}}\right|_{X}\right)_{t \geq 0}\right\rangle$ be a uniformly bounded empathy. Then A satisfies the Hille-Yosida estimates in $X$, and consequently

(1) $\left(\left.e^{t \mathfrak{A}}\right|_{\overline{D(A)}}\right)_{t \geq 0}$ is a $C_{0}$-semigroup in $\overline{D(A)}$,

(2) if $X$ has the Radon-Nikodym property, then additionally $\left(\left.e^{t \mathfrak{A}}\right|_{X}\right)_{t>0}$ is a bounded strongly continuous semigroup of bounded operators in $X$ (but in general not a $C_{0}$-semigroup),

(3) if $X$ is reflexive or $\overline{D(A)}=X$, then $\left(e^{t \mathfrak{A}_{\mid}}\right)_{t \geq 0}$ is a $C_{0}$-semigroup generated by $A$.

Proof. If $\left\langle(Y(t))_{t \geq 0},\left(\left.e^{t \mathfrak{A}}\right|_{X}\right)_{t \geq 0}\right\rangle$ is a uniformly bounded empathy, then by [19, Theorem 7.1] there is $N$ such that for any $\lambda>0, k=1,2, \ldots$ we have $\left\|\lambda^{k} R^{k}(\lambda)\right\| \leq N$. From (3.33) we obtain that

$$
\left\|(\lambda I-A)^{-k}\right\| \leq \lambda^{-k} N,
$$

hence $A$ satisfies the Hille-Yosida estimates in $X$. The rest of the proof follows from the well-known theorem by Arendt [2] (see also [5]).

Another avenue to explore is based on the following simple observation which follows immediately from the definition of $B$-bounded semigroups.

Proposition 3.12. The pair $\left\langle(Y(t))_{t \geq 0},(\exp (t \mathfrak{A}))_{t \geq 0}\right\rangle$ is an empathy in the pair $Z, X_{B}$.

This proposition suggests that if one could find a space $X^{\prime} \subset X$ such that $X$ is a completion of $X^{\prime}$ with respect to the norm $\left\|K^{-1} \cdot\right\|_{Z}$, then the notion of $B$-bounded semigroup generated in the pair $X^{\prime}, Z$ would coincide with the notion of empathy in the pair of spaces $X, Z$. However, the following result shows that also here the choice is very limited.

Proposition 3.13. The pair $\left\langle(Y(t))_{t \geq 0},(\exp (t \mathfrak{A}))_{t \geq 0}\right\rangle$ is an empathy in $Z, X$ if and only if $K$ is a bounded operator, with a bounded densely defined inverse $K^{-1}$.

Proof. The pair $\left\langle(Y(t))_{t \geq 0},(\exp (t \mathfrak{A}))_{t \geq 0}\right\rangle$ is an empathy in $Z, X$ if and only if $X_{B}$ can be identified with $X$. By Theorem 2.8 this is possible if and only if $B=K^{-1}$ is closeable and $B^{-1}=K$ is bounded. $X_{B}$ is then identified with $D(\bar{B})=D\left(\overline{K^{-1}}\right)$ and by Theorem 2.8 again we must have $D\left(\overline{K^{-1}}\right)=X$. 
Next, the operator $\overline{K^{-1}}$ is invertible (because it equals $\mathfrak{B}$ ). It follows that $\bar{K}=$ $\left(\overline{K^{-1}}\right)^{-1}$. In fact, $y=\bar{K} x$ if and only if $x=\lim _{n \rightarrow \infty} x_{n}, x_{n} \in D(K)$ and $y=$ $\lim _{n \rightarrow \infty} K x_{n}$. Putting $y_{n}=K x_{n}$ we obtain that this is equivalent to $\lim _{n \rightarrow \infty} y_{n}=y$, $y_{n} \in D\left(K^{-1}\right)$ and $\lim _{n \rightarrow \infty} K^{-1} y_{n}=x$, thus $\overline{K^{-1}} y=x$ which due to the invertibility of $\overline{K^{-1}}$ proves the statement. Since for bounded operators the closure is equal to the extension by continuity, denoted for a moment by $\tilde{K}$, we obtain that

$$
\tilde{K}: \overline{D(K)} \stackrel{\text { onto }}{\longrightarrow} X
$$

is invertible and bounded operator. Since $\overline{D(K)}$ is a Banach space, $\tilde{K}^{-1}=\overline{K^{-1}}$ is a bounded operator and clearly $K^{-1}$ is bounded. Using again the fact that the closure of a bounded operator is equal to its extension by continuity, we obtain that $X=D\left(\overline{K^{-1}}\right)=$ $\overline{D\left(K^{-1}\right)}=\overline{\operatorname{Im} K}$.

The proof in the reverse direction is obvious.

It is instructive to compare Hille-Yosida type estimates required for generation of empathy and $B$-bounded semigroup. For simplicity, we assume that the estimates are valid with $k=1$ and $N=1$ for the empathy and with $k=1, M=1, \omega=0$ for $B$-bounded semigroup. In such a case equations (3.28) can be transformed to

$$
\begin{gathered}
\left\|\left(\lambda I-K^{-1} L\right)^{-1} f\right\|_{Z} \leq \lambda^{-1}\|K f\|_{X}, \quad f \in D(K) \subset Z, \\
\left\|\left(\lambda I-L K^{-1}\right)^{-1} u\right\|_{X} \leq \lambda^{-1}\|u\|_{X} .
\end{gathered}
$$

The second inequality is simply the Hille-Yosida estimate for the operator $L K^{-1}$ in $X$ (and thus if $K$ and $L$ commute and $X=Z$, this condition is equivalent to (3.23)).

On the other hand, for the generation a $K^{-1}$-bounded semigroup of contractions we must have by, Theorem 3.4,

$$
\left\|\left(\lambda I-K^{-1} L\right)^{-1} x\right\|_{Z} \leq \lambda^{-1}\|x\|_{Z} \quad \forall x \in D(K) \subset Z .
$$

Summarizing, for the generation of $K^{-1}$-bounded semigroup of contractions, the operator $K^{-1} L$ must generate a semigroup of contractions in $Z$. On the other hand, for generation of an empathy, we must have a generation of a semigroup of contractions in $X$ by $L K^{-1}$ and additionally, the resolvent of $K^{-1} L$ must be a continuous operator from $D(K)$ with graph norm to $Z$. Explanation of the different order in which the operators $K^{-1}$ and $L$ enter the Hille-Yosida estimates comes from Proposition 3.12. If we think of empathy as a $B$-bounded semigroup with $X=X_{B}^{\prime}$, then the estimate (3.38) follows directly from (2.9). Now the estimate (3.39) is obtained from (3.15) by passing from $X$ to a new space $X_{B}$. See also Subsection 3.4 where we discuss a suitable example.

3.3. Showalter's approach. The approach presented in [20] is very similar in spirit to the ideas leading to the construction of a $B$-bounded semigroup. The theory presented there covers, however, much broader class of problems including nonlinear evolution inclusions. On the other hand, a number of assumptions on $K$ are more restrictive than 
those appearing in the theory of $B$-bounded semigroups. For simplicity we outline here the approach of [20] in the case of linear evolution equations.

It is assumed that $K: E \rightarrow E^{*}$ is a linear, symmetric, and nonnegative operator from a linear space $E$ to its algebraic dual. This allows us to introduce a semi-scalar product on $E$; the corresponding semi-normed space is denoted by $E_{b}$. Its continuous dual $E_{b}^{\prime}$ is a Hilbert space. The operator $L$ is assumed to act from a domain in $E_{b}$ into $E_{b}^{\prime}$.

In a particular case when $K$ generates a scalar product under which $E_{b}$ is complete, $K$ becomes the Riesz isomorphism onto $E_{b}^{\prime}$. Thanks to this, one can proceed as in Theorem 3.2 converting problem (1.1) into the standard Cauchy problem

$$
\frac{d u}{d t}=K^{-1} L u, \quad \lim _{t \rightarrow 0^{+}} u(t)=K^{-1} \stackrel{\circ}{u}
$$

in $E_{b}$. Hence, the question to be solved is whether $K^{-1} L$ generates a semigroup in $E_{b}$.

Using the $B$-bounded semigroup approach we obtain the same problem under the following particular assumptions. Let $D(K) \subset X$ and $(K, D(K))$ be a symmetric, positive operator in $X$. Assume that $E_{b}$ is the completion of $D(K)$ under the scalar product $(K x, y)$; then its continuous dual $E_{b}^{\prime}$ is exactly the space $X_{B}\left(B=K^{-1}\right)$ as $K$ is the Riesz isomorphism between $E_{b}$ and $E_{b}^{\prime}$.

Here we see that $B$-bounded semigroups offer a greater flexibility, as, for example, if we keep $Z=X$, then as $X_{B}$ we obtain a space such that a suitable extension of $K$ is an isomorphism between $\overline{D(K)}^{X}$ and $X_{B}$. We will see an example of this kind in the next subsection.

The approach of [20] has been used also when $K$ is not invertible. Again, the idea is similar to that used in the construction of $B$-bounded semigroup with noninvertible $B$-one passes to the quotient space $D(K) / N(K)$. The problem is that the second operator involved, $L$, not necessarily can be made to act in such a quotient space. In $B$-bounded semigroups theory the properties of $B$-bounded semigroups ensured that this was indeed possible. In [20] the author introduced a suitable multivalued relation which fortunately preserves the essential properties of the original operator. This seems to be a complementary approach to that sketched in Remark 3.8, but the comparison of these methods is still an open question.

3.4. The Sobolev-Galpern equation. Consider a model problem which can be dealt with using all three approaches:

$$
\partial_{t}(\Delta u)=\Delta^{2} u,\left.\quad u\right|_{\partial \Omega}=0,\left.\quad \partial_{\nu} u\right|_{\partial \Omega}=0,\left.\quad \Delta u\right|_{t=0}=\stackrel{\circ}{u},
$$

where $\Omega \subset \mathbb{R}^{n}$ is a bounded sufficiently smooth set.

The empathy theory (see [17] where, in fact, an early version of empathy theory, called the $B$-evolution theory was used), the solution operators $t \rightarrow S(t)$ act from $L_{2}(\Omega)$ into $W_{2}^{4}(\Omega) \cap \stackrel{\circ}{W} \underset{2}{2}(\Omega)$, the related semigroup $(E(t))_{t \geq 0}$, generated by a suitable realization of $L K^{-1}=\Delta^{2} \Delta^{-1}$ acts in $L_{2}(\Omega)$ and the equation is also satisfied in $L_{2}(\Omega)$.

Using Showalter's approach we obtain $E_{b}=\stackrel{\circ}{W} \underset{2}{1}(\Omega)$ with $E_{b}^{\prime}=W_{2}^{-1}(\Omega)$; it follows that the solution is a $\stackrel{\circ}{W}_{2}^{1}(\Omega)$-valued function and the equation is satisfied in $W_{2}^{-1}(\Omega)$. 
Using $B$-bounded semigroups we are able to construct even weaker solutions. Let $Z=X=L_{2}(\Omega), K=\Delta$ with $D(K)=W_{2}^{2}(\Omega) \cap \stackrel{\circ}{W}_{2}^{1}(\Omega)$ and $L=\Delta^{2}$ with $D(L)=$ $W_{2}^{4}(\Omega) \cap \stackrel{\circ}{W}_{2}^{2}(\Omega)$. From the general theory of PDE's we know that $K$ is an isomorphism onto $L_{2}(\Omega)$. From the elliptic regularity it follows that $X_{B}=\left(W_{2}^{2}(\Omega) \cap \stackrel{\circ}{W_{2}^{1}}(\Omega)\right)^{\prime}$ (the continuous dual). Since all the occurring spaces are reflexive, we have to check only the Hille-Yosida estimates. Let $u \in D(L)$, then we have

$$
\left(\lambda u-K^{-1} L u, u\right)_{X}=\lambda\|u\|_{X}^{2}-\left(K^{-1} L u, u\right)_{X},
$$

next

$$
\begin{aligned}
\left(K^{-1} L u, u\right)_{X} & =\left(L K K^{-1} u, K^{-1} u\right)_{X}=(L K f, f)_{X} \\
& =\int_{\Omega} \Delta^{2} \Delta f \bar{f} d x=-\int_{\Omega}|\nabla \Delta f|^{2} d x \leq 0,
\end{aligned}
$$

hence

$$
\lambda\|u\|_{X}^{2} \leq\left(\lambda u-K^{-1} L u, u\right)_{X} \leq\left\|\lambda u-K^{-1} L u\right\|_{X}\|u\|_{X} .
$$

Now, let $x \in D(K)$ and consider the equation $x=\lambda u-K^{-1} L u$. We can transform this equation to

$$
K x=\lambda K u-L u
$$

with $K x \in X$. Since we have

$$
(K u, u)_{X} \geq c_{1}\|u\|_{1}^{2}, \quad(-L u, u)_{X} \geq c_{2}\|u\|_{2}^{2},
$$

we obtain for $u \in \stackrel{\circ}{W} \underset{2}{ } \subset D(K)$

$$
(\lambda K u-L u, u) \geq c_{1}\|u\|_{1}^{2}+c_{2}\|u\|_{2}^{2} \geq c_{2}\|u\|_{2}^{2},
$$

hence $\lambda K-L$ is coercive on $\stackrel{\circ}{W}_{2}^{2}$ and for each $K x \in X$ there is $u \in \stackrel{\circ}{W}_{2}^{2}$ (in fact, in $D(L)$ from the elliptic regularity) satisfying (3.45). Thus, we see that the Hille-Yosida estimates (3.23) is satisfied and problem (3.41) has a unique $X_{B}$-solution.

It is interesting to note that in this case also the first two solutions can be obtained by $B$-bounded semigroup method by a suitable choice of the space $X$.

\section{Acknowledgement}

This work was supported by the Foundation for Research and Development of South Africa and the University of Natal Research Fund.

\section{References}

[1] W. Arendt, Resolvent positive operators, Proc. London Math. Soc. (3) 54 (1987), no. 2, 321-349. MR 88c:47074. Zbl 617.47029.

[2] _ Vector-valued Laplace transforms and Cauchy problems, Israel J. Math. 59 (1987), no. 3, 327-352. MR 89a:47064. Zbl 637.44001.

[3] L. Arlotti, On B-bounded semigroups as a generalization of $C_{0}$-semigroups, Z. Anal. Anwendungen 19 (2000), no. 1, 23-34. CMP 1748056. 
[4] J. Banasiak, Generation results for B-bounded semigroups, Ann. Mat. Pura Appl. (4) 175 (1998), 307-326. CMP 1748 230. Zbl 991.53697.

[5] J. Banasiak and V. Singh, B-bounded semigroups and C-existence families, Taiwanese J. Math. 5 (2001), no. 4, in print.

[6] L. Bartoli and S. Totaro, Approximation of B-bounded semigroups, Adv. Math. Sci. Appl. 7 (1997), no. 2, 579-600. MR 98k:47075. Zbl 903.47022.

[7] B. Bäumer and F. Neubrander, Relatively closed operators and linear evolution equations, Louisiana State Univ. Seminar Notes in Functional Analysis and PDEs, Baton Rouge, 1993.

[8] A. Belleni-Morante, B-bounded semigroups and applications, Ann. Mat. Pura Appl. (4) 170 (1996), 359-376. MR 98c:47049. Zbl 882.47012.

[9] _ On some properties of B-quasi bounded semigroups and applications, Quaderno sezione modelli matematici, no. 1, Dipartimento di Ingegneria Civile, Universitá di Firenze, 1996.

[10] A. Belleni-Morante and S. Totaro, The successive reflection method in three-dimensional particle transport, J. Math. Phys. 37 (1996), no. 6, 2815-2823. MR 97h:82059. Zbl 892.47065.

[11] A. Favini and P. Plazzi, On some abstract degenerate problems of parabolic type. I. The linear case, Nonlinear Anal. 12 (1988), no. 10, 1017-1027. MR 90b:34095a. Zbl 707.35080.

[12] A. Favini and A. Yagi, Multivalued linear operators and degenerate evolution equations, Ann. Mat. Pura Appl. (4) 163 (1993), 353-384. MR 94f:34116. Zbl 786.47037.

[13] A. Haraux, Linear semigroups in Banach spaces, Semigroups, Theory and Applications, vol. II (Trieste, 1984), Pitman Res. Notes Math. Ser., vol. 152, Longman Sci. Tech., Harlow, 1986, pp. 93-135. MR 88c:47078. Zbl 619.47034.

[14] C. Knuckles and F. Neubrander, Remarks on the Cauchy problem for multi-valued linear operators, Partial Differential Equations (Han-sur-Lesse, 1993) (Guenter Lumer, ed.), Math. Res., vol. 82, Akademie-Verlag, Berlin, 1994, Models in Physics and Biology, pp. 174-187. MR 96f:34079. Zbl 823.34062.

[15] I. V. Mel'nikova, General theory of the ill-posed Cauchy problem, J. Inverse Ill-Posed Probl. 3 (1995), no. 2, 149-171. MR 97a:34156. Zbl 835.35148.

[16] I. V. Mel'nikova and M. A. Al'shansky, Well-posedness of the Cauchy problem in a Banach space: regular and degenerate cases, J. Math. Sci. (New York) 87 (1997), no. 4, 3732 3780. MR 99a:34162. Zbl 922.34050.

[17] N. Sauer, Linear evolution equations in two Banach spaces, Proc. Roy. Soc. Edinburgh Sect. A 91 (1981/82), no. 3-4, 287-303. MR 84h:34099. Zbl 529.47028.

[18] __ Implicit evolution equations and empathy theory, Recent Developments in Evolution Equations (Glasgow, 1994) (A. C. McBride and G. F. Roach, eds.), Pitman Res. Notes Math. Ser., vol. 324, Longman Sci. Tech., Harlow, 1995, pp. 32-39. MR 97h:34078. Zbl 835.47031.

[19] _ Empathy theory and the Laplace transform, Linear Operators (Warsaw, 1994) (Jan Janas et al., eds.), Banach Center Publ., vol. 38, Polish Acad. Sci., Warsaw, 1997, pp. 325338. MR 98e:47062. Zbl 885.47013.

[20] R. E. Showalter, Monotone Operators in Banach Space and Nonlinear Partial Differential Equations, Mathematical Surveys and Monographs, vol. 49, American Mathematical Society, Rhode Island, 1997. MR 98c:47076. Zbl 870.35004.

[21] E. Zeidler, Nonlinear Functional Analysis and its Applications. I, Springer-Verlag, New York, 1986, Fixed-point theorems. Translated from the German by Peter R. Wadsack. MR 87f:47083. Zbl 583.47050.

J. Banasiak: Department of Mathematics and Applied Mathematics, University of Natal, Durban 4041, South Africa 


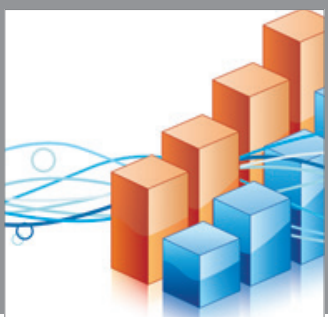

Advances in

Operations Research

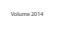

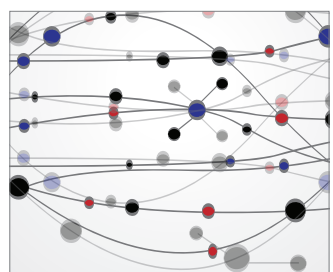

\section{The Scientific} World Journal
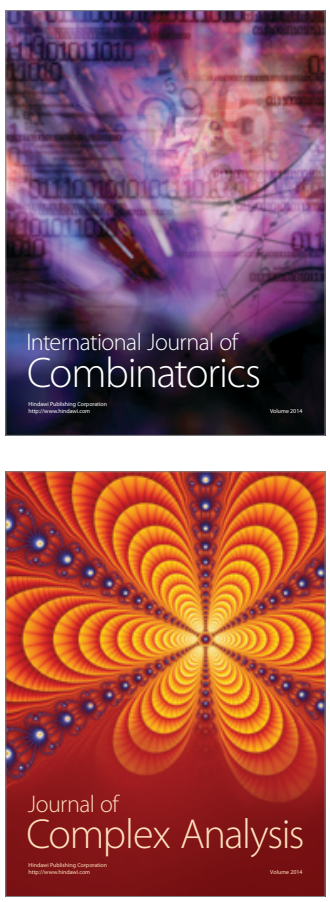

International Journal of

Mathematics and

Mathematical

Sciences
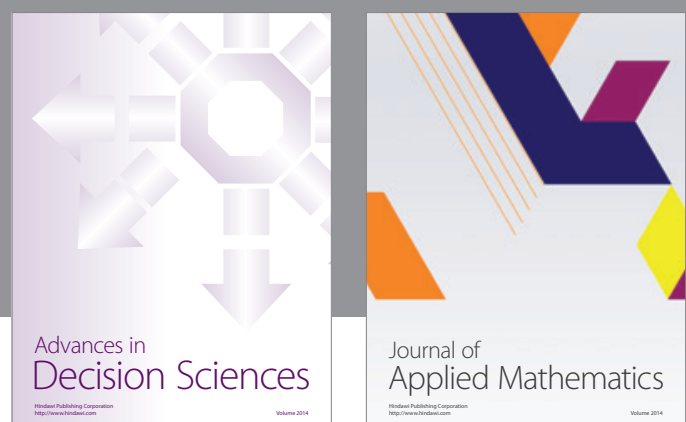

Journal of

Applied Mathematics
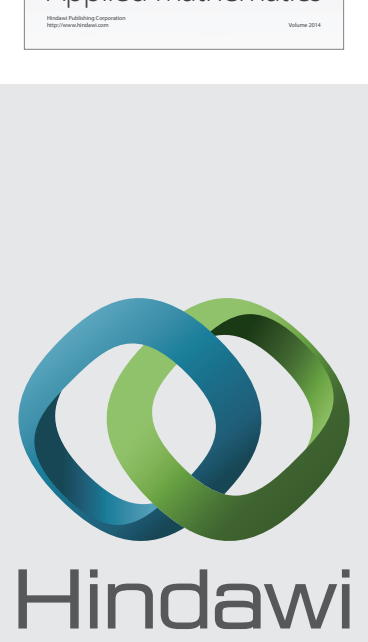

Submit your manuscripts at http://www.hindawi.com
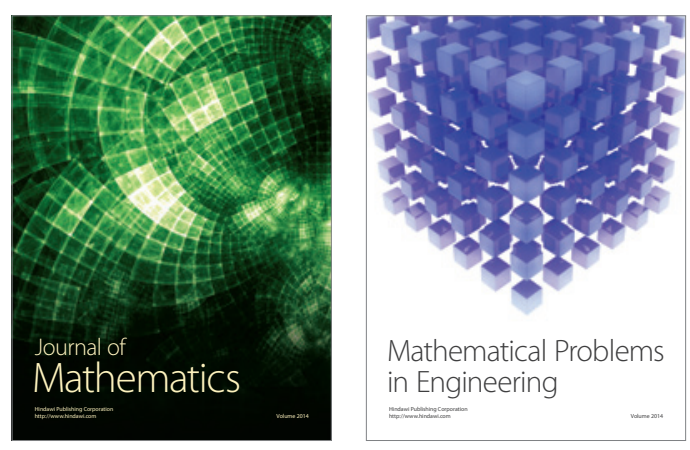

Mathematical Problems in Engineering
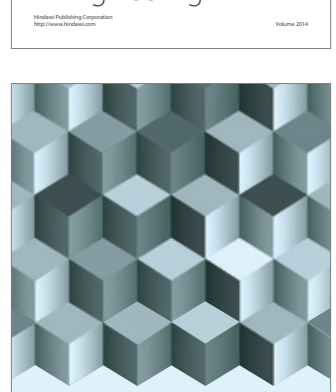

Journal of

Function Spaces
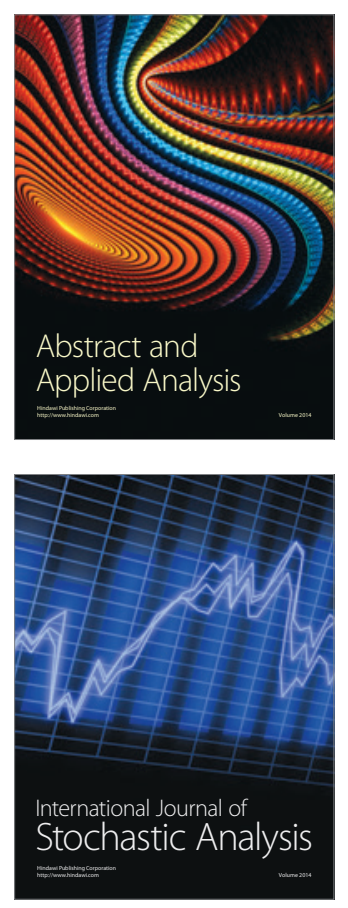

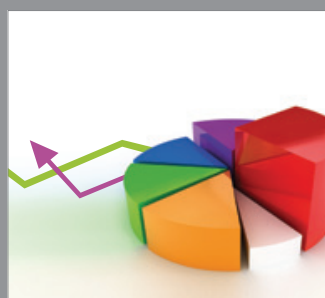

ournal of

Probability and Statistics

Promensencen
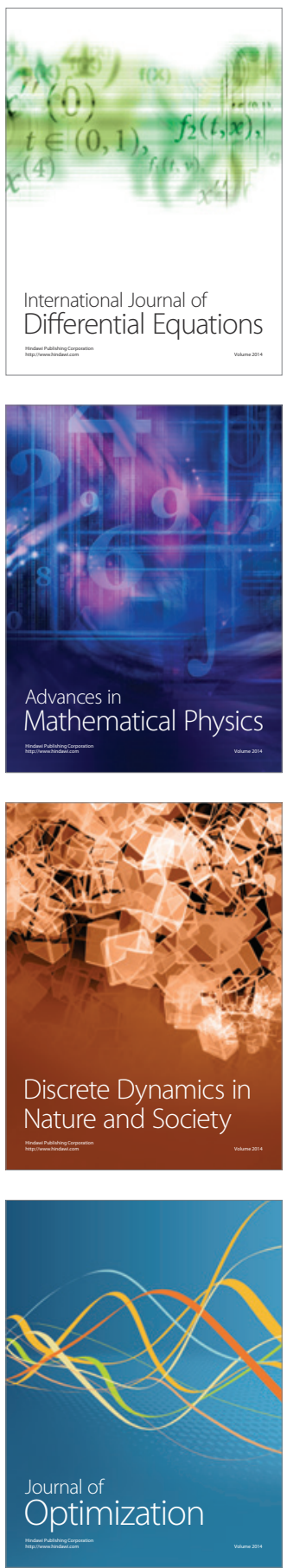